\title{
Research Trends in Biochemical Analysis
}

\section{Edward P.C. Lai*}

Department of Chemistry, Carleton University, Ottawa, Ontario, Canada

Biochemistry is at the heart of life science dealing with the chemistry of biological processes. Current research attempts to utilize the principles of chemistry for elucidation of the living system, in combating serious disease in humans, animals and plants. Biochemical analysis focuses on the chemical composition of a particular living system or biological substance using various experimental techniques and computational simulations [1,2]. A survey of the latest research trends is summarized below to highlight many interesting findings from the last several months.

Post-mortem investigation of the final cause of death involves biochemical analysis of the vitreous humor from the eye. A range of analytes can be tested on modern biochemical analyzers. However, it is not clear which analytes should be requested in order to prevent unnecessary testing and expense. Mitchell and coworkers reviewed the contribution of each analyte in post-mortem contexts to reduce vitreous biochemistry test requests for sodium, osmolality, glucose, ketones ( $\beta$-hydroxybutyrate), urea and creatinine [3]. Critical appraisal of each part of the post-mortem process should be undertaken to provide evidence to justify any investigative methods used in an autopsy. Sensitization prevalence to moulds reached from less than $10 \%$ in the general population to more than $25 \%$ in asthmatic and/or atopic subjects. Skin Prick Test (SPT) and specific IgE (sIgE) measurement are normally recommended to diagnose IgE-mediated mould sensitization. More often than not, agreement between SPT and sIgE results is less than $50 \%$ and standardization of the extracts is required to achieve reliable test results. Kespohl and co-workers analyzed mould SPT extracts of Alternaria alternata, Aspergillus fumigatus, Cladosporium herbarum and Penicillium chrysogenum from six manufacturers [4]. Protein-, antigen- and IgE-binding contents were quantified by Bradford assay, sandwich ELISA and IgE-ImmunoCAP-inhibition tests. Protein composition and IgE and IgG binding were analyzed by SDS-PAGE and immunoblotting, respectively. With respect to quantity and quality of protein, antigen and human IgE-binding content, those commercially available mould SPT extracts showed high variability raising the question of comparability and reliability of SPT results.

Banana lignocellulosic biomass that is abandoned after fruit harvesting has biochemical methane potential as an energy source. Kamdem and co-workers monitored volatile fatty acid biogas production by $\mathrm{KOH}$ from six morphological parts of the banana cultivar using high performance liquid chromatography and gas chromatography [5]. The bulbs, leaf sheaths, petioles-midribs, leaf blades, rachis stems, and floral stalks gave total biogas production of $126-256 \mathrm{ml} \mathrm{g}^{-1}$ dry matter and total biomethane production of 98$162 \mathrm{ml} \mathrm{g}^{-1}$. The biogas production rates and yields depended on the biochemical composition of the biomass and the ability of anaerobic microbes to access fermentable substrates. Acetate was the major VFA in all the MP sample culture media. The bioconversion yields for each morphological part were below $50 \%$, showing that these substrates were not fully biodegraded after 188 days. The estimated electricity that could be produced from biogas combustion after fermenting all of the biomass produced annually by the Del Monte plantations for 188 days is approximately $10^{7} \mathrm{~kW} \mathrm{~h}$, which would be worth one million euros in the current market and could serve over 40,000 people in the region.
Biochemical conversion of lignocellulose to fermentable carbohydrates for ethanol production is now being implemented in large-scale industrial production. The conversion process generates a residue containing substantial amounts of lignin, which is mainly incinerated for heat and power generation. Hansen and co-workers analyzed the structural and chemical composition of the solid and liquid fractions of lignin residue from wheat straw [6]. Roughly 70 and $15 \%$ of the solid mass fraction consisted of lignin and ash, respectively. Residual carbohydrates mostly originated from hemicellulose in the liquid fraction and from cellulose in the solid fraction. The solid fraction also contained significant amounts of protein, which is a valuable byproduct when used as animal feed or when enzymes and yeast cells are separated for process recycling. Silica was the dominant constituent in the mineral fraction and most particles in the solid fraction appeared as silica coated by lignin, hampering separation of the two components before incineration or refinement of the residue. Kang and co-workers estimated biogas production by anaerobic digestion of agricultural byproduct silage from hot pepper farms [7]. Biochemical tests showed that the ultimate methane potential of ensiled material was higher than that of raw material without silage storage, with a lower firstorder hydrolysis constant. In comparison with raw material and silage material without additives, silage materials containing microbial additives all showed higher ultimate methane potentials and first order hydrolysis constants, with the highest values found on day 40 .

Arsenic contamination in the environment has attracted considerable attention worldwide. Ahmad and Gupta compared the effect of $\mathrm{As}(\mathrm{III})$ and $\mathrm{As}(\mathrm{V})$ species on accumulation, biochemical responses, and gene expression in Brassica juncea var. Pusa Jaganath [8]. Hydroponically grown 14-day-old seedlings of B. juncea were treated with different concentrations of $\mathrm{As}(\mathrm{III})$ and $\mathrm{As}(\mathrm{V})$. Accumulation of total As increased with increasing concentration of both As species and exposure time, mainly in roots. Reduction in seed germination, root-shoot length, chlorophyll, and protein content were observed more in As(III)-treated leaves. Antioxidant enzymes and stress-related parameters (cysteine, proline, and malondialdehyde) were stimulated, allowing plant to tolerate both As species. Sodium Dodecyl Sulfate-Polyacrylamide Gel Electrophoresis (SDS-PAGE) analysis of the leaves showed significant changes in protein profile with more stringent effect with As(III) stress. Semiquantitative RT-PCR analysis showed regulation in expression of phytochelatin synthase, metallothionine-2, glutathione reductase, and glutathione synthetase

*Corresponding author: Edward P.C. Lai, Department of Chemistry, Carleton University, Ottawa, Ontario, Canada, Tel: 613-520-2600; E-mail: edwardpclai@gmail.com

Received November 25, 2013; Accepted November 27, 2013; Published November 29, 2013

Citation: Lai EPC (2013) Research Trends in Biochemical Analysis. Biochem Anal Biochem 2: e144. doi: 10.4172/2161-1009.1000e144

Copyright: (c) 2013 Lai EPC. This is an open-access article distributed under the terms of the Creative Commons Attribution License, which permits unrestricted use, distribution, and reproduction in any medium, provided the original author and source are credited. 
genes. All results suggested that accumulation and inhibition on physiological parameters differ according to the As species, while molecular and biochemical parameters showed a combinatorial type of tolerance mechanism against $\mathrm{As}(\mathrm{III})$ and $\mathrm{As}(\mathrm{V})$ stresses.

Microbial utilization is a key transformation process of soil organic matter. Apostel and co-workers traced the metabolites of two amino acids in microbial groups by combining position-specific $13 \mathrm{C}$ labeling with compound-specific 13C-PLFA analysis [9]. The fate of individual $\mathrm{C}$ atoms was analyzed by position-specific labeling to gain insight into the mechanisms and kinetics of microbial utilization. Discrimination between positions is highest in the initial reactions and decreases with time. Microbial transformation pathways were constructed to show that the $\mathrm{C}_{2}$ position of alanine is lost faster than its $\mathrm{C}_{3}$ position either anabolically or catabolically. Only two out of eight microbial groups incorporated glutamic acid $\mathrm{C}_{2}$ into PLFAs. It is therefore a promising metabolic tracer in regard to ecophysiology of cells and therefore changing environmental conditions. This approach strongly improves our understanding of soil C fluxes. Oats (Avena spp.) produce antimicrobial compounds (avenacins) that provide protection against disease. Avenacins are synthesized from the simple triterpene, $\beta$-amyrin. Geislera and co-workers carried out functional analysis of the oat enzyme AsCYP51H10 which is a divergent member of the CYP51 cytochrome P450 family [10]. They showed that both hydroxylation and epoxidation of $\beta$-amyrin (to give $12,13 \beta$-epoxy-16 $\beta$ hydroxy- $\beta$-amyrin) were catalyzed by this enzyme and that the C12,13 epoxy group has antifungal activity. This critical discovery of triterpene metabolic engineering has important applications for biotechnology in food, health, and industry.

Glycogen storage disease type II can result from genetic deficiency of Glucosidase Alpha Acid (GAA). To Martiniuk and co-workers investigated whether a functional recombinant human GAA enzyme (tobrhGAA) could be generated in tobacco seeds for future enzyme replacement therapy [11]. Human GAA cDNA was subcloned into the plant expression plasmid-pBI101 under the control of the soybean $\beta$-conglycinin seed-specific promoter. Tobacco seeds contain the metabolic machinery that is more compatible with mammalian glycosylation-phosphorylation and processing. They found that tobrhGAA was readily taken up by human GSDII fibroblast cells. The tobrhGAA corrected the enzyme defect in tissues at 7 days after a single dose following Intraperitoneal (IP) administration in GAA knockout mice. Demonstrably, tobrhGAA contained the minimal phosphorylation and mannose-6-phosphate residues essential for biological activity. Mikashinovich and Belousova demonstrated that hypoxia and imbalance in the antioxidant enzyme system contributed to the development of side effects of statins [12]. An increase in glycolysis metabolite concentrations and glucose-6-phosphate dehydrogenase activity was revealed by their analysis of metabolic changes in rat erythrocytes.

Membrane-bound proteases play important roles in protein quality control and various regulatory functions. A catalytic SerLys dyad in the N-terminal region of PH1510p (1510-N) is a serine protease that specifically cleaves the p-stomatin PH1511p at the C-terminal hydrophobic region. Yokoyama and co-workers performed heat treatment of the protease-peptide complex before crystallization in order to better understand the catalytic mechanism of 1510-N [13]. Between the structures with heat treatment and with no heat treatment, the C-terminal half of the peptide is slightly deviated even though the $\mathrm{N}$-terminal half of the peptide (which binds to $1510-\mathrm{N}$ more tightly than the C-terminal half) is well superposed. The hydrolase fold is one of the most versatile structures in the protein realm according to the diversity of sequences adopting such a three-dimensional architecture. Benavente and co-workers determined the biochemical characteristics of Cest-2923 which is a carboxylesterase from the lactic acid bacterium Lactobacillus plantarum WCFS1 [14]. The crystal structure of Cest2923 has a basic framework of canonical $\alpha / \beta$ hydrolase fold. Its monomers, canonical dimers and tetramers (commonly found in the hormone-sensitive lipase family) exhibit $\mathrm{pH}$-dependent pleomorphic behaviour in solution. Interaction with the putative oxyanion hole was identified by the presence of sulfate or acetate molecules in the close vicinity of the nucleophile Ser116, hence deducing the existence of hydrolytic activity within Cest-2923 crystals.

A novel pectate lyase 3 (Bx-PEL3) gene from the stage-specifically expressed sequence tag library of Bursaphelenchus xylophilus was cloned by Lee and co-workers [15]. Recombinant Bx-PEL3 showed activity against polygalacturonic acid. It was structurally similar to PEL from the Bacillus strain KSM P-15 and shared essential residues for calcium binding and catalytic activity. Bx-PEL3 was expressed near the esophageal gland and might be involved in the cell wall degradation as a biochemically functional pathogenic factor. Chen and co-workers evaluated the risk of Biochemical Recurrence (BCR), hypermethylation of genes, and Glutathione-S-Transferase (GST) polymorphisms in relation to prostate cancer [16]. For patients after prostatectomy or radiotherapy, accurate prediction of BCR is critical. GST polymorphisms, hypermethylation of GSTP1 and functional genes in carcinogenesis (including tumor suppression gene and differentiation gene) were reported to be associated with $\mathrm{BCR}$. Intermediate end points are desirable to expedite the integration of neoadjuvant systemic therapy into the treatment strategy for high-risk localized prostate cancer. Galsky and co-workers analyzed the correlation between endorectal magnetic resonance imaging at $1.5 \mathrm{Tesla}$ ( $1.5 \mathrm{~T}$ erMRI) response to neoadjuvant chemotherapy and biochemical recurrence in patients with high-risk localized prostate cancer [17]. Trial 1 explored docetaxel for 6 months and Trial 2 explored docetaxel plus bevacizumab for 4.5 months, both before radical prostatectomy. Among the 53 evaluable patients in the combined analysis, 33\% achieved a Prostate-Specific Antigen (PSA) response, 27\% achieved an erMRI partial response and $40 \%$ achieved an erMRI minor response. Median follow-up was 4.2 years, and $62 \%$ of the evaluable patients developed biochemical recurrence. These data do not support the use of 1.5T erMRI response as a primary end point in neoadjuvant chemotherapy trials.

The cell cytoskeleton is a dynamic signaling scaffold that transmits complex mechanosensing stimuli that regulate normal physiological and aberrant pathophysiological processes. Choi and co-workers developed a purification method that enriches for the cytoskeleton and its associated proteins in their native state that is also compatible with current mass spectrometry-based protein detection methods [18]. This method can be used for large-scale biochemical and proteomic analyses of different cell types. In the purified mouse embryonic fibroblasts, 635 cytoskeleton or cytoskeleton-interacting proteins were identified. Important nodes of interconnectivity surrounding well-established regulators of the actin cytoskeleton and focal adhesion complexes were revealed by functional annotation and network analyses. This improved cytoskeleton purification method will aid our understanding of how the cytoskeleton controls normal and diseased cell functions. Escribano and co-workers studied the reference values of different haematological and biochemical parameters in the Spanish Purebred horse (SPB, Andalusian) in each of the stages of a programmed exercise 
on a treadmill system [19]. Ninety four young SPB male horses (4.2 \pm 2.3 years old) were used. An increasing intensity exercise test at 4, 5, 6 and $7 \mathrm{~m} / \mathrm{s}$ was carried out on a treadmill. Total red blood cells, total white blood cells, neutrophils and lymphocytes counts; haematocrit, haemoglobin, lactate, uric acid, creatinine, total plasma protein concentrations, and aspartate transaminase, lactate dehydrogenase, and creatine-quinase activities were determined. In conclusion, (i) the reference values for each parameter were determined for each of the exercise tests, (ii) medium-high heritability and a high repeatability were manifested by all the parameters analysed. Measuring guidelines for improving the athletic ability of SPB horses were determined, for selecting these animals in response to those parameters and for establishing the heritability as well as genetic correlations.

Pantazis and co-workers developed a sensitivity analysis methodology suitable for complex stochastic reaction networks with a large number of parameters [20]. Their information theory approach relied on the quantification of information loss due to parameter perturbations. They employed the rigorously-derived relative entropy rate, which is directly computable from the propensity functions, in the sensitivity analysis. The Fisher Information Matrix (FIM) revealed hidden parameter dependencies and sensitivities in reaction networks. The method was validated on three biochemical systems: (a) a protein production/degradation model where explicit solutions are available, (b) the p53 reaction network where quasi-steady stochastic oscillations are observed between high and low populations, and (c) an epidermal growth factor receptor model with more than 200 reactions and corresponding parameters. Fluorescent double-stranded DNA (dsDNA) molecules are commonly produced from complementary single-stranded DNA (ssDNA) molecules, labeled with fluorescent dyes at the same $\left(3^{\prime}\right.$ or $\left.5^{\prime}\right)$ end, by annealing. For quantitative biochemistry, accurate measurement of the percentage of doubly labeled dsDNAs is a critical prerequisite that has puzzled scientists for decades. Hou and coworkers established a Fluorescence Correlation Spectroscopy (FCS) system to measure the percentage of doubly labeled dsDNAs (PDL) in the total dsDNA pool [21]. The method requires a concentration of DNA in the nano-molar range and a sample volume of tens of microliters.

Fatima and co-workers produced non-embryogenic, synthetic seeds by encapsulating nodal segments (containing axillary buds) of Withania somnifera $\mathrm{L}$. in calcium alginate hydrogel containing Murashige and Skoog (MS) medium [22]. Sodium alginate (3\%) with calcium chloride $(100 \mathrm{mM})$ was found to be the optimum concentration for the production of uniform synthetic seeds. Effect of different treatments with MS medium containing different concentrations of cytokinins $(0.5,1.0,2.5,5.0 \& 10.0 \mu \mathrm{M})$ along with $\alpha$-naphthalene acetic acid $(0.5$ $\mu \mathrm{M})$ on in vitro morphogenic response of synthetic seeds was evaluated. Maximum frequency (86\%) of conversion of encapsulated beads into plantlets was achieved on a MS medium containing 6-benzyladenine and 6-benzyladenine $(2.5 \mu \mathrm{M})$ after 4 weeks of culture. The plantlets were hardened, acclimatized and grew well in the field without any detectable malformation. Significant enhancement in the pigment contents and net photosynthetic rates with increasing acclimatization days might be attributed to chlorophyll biosynthesis.

Activities of antioxidant enzymes (superoxide dismutase, catalase and peroxidase) were significantly increased, suggesting their preventive role in membrane oxidation and damage to biological molecules. An enhanced level of lipid peroxidation, as indicated by malondialdehyde content, clearly indicated its positive role in combating oxidative stress during acclimatization of plantlets. Their random amplified polymorphic DNA and inter-simple sequence repeat profiles confirmed the genetic stability among the clones. This synthetic seed technology could pave the way for conservation with potential storability and limited quarantine restrictions. Expansins cause plant cell wall loosening and are present primarily in the plant kingdom. Georgelis and co-workers provide evidence that expansins from three plant pathogenic bacteria and one fungus cause extension of cell walls in vitro and weaken cellulose filter paper networks, without lytic activity [23]. However, those microbial expansins did not synergistically enhance the activity of several cellulases in hydrolysis of cellulose during plant pathogenesis.

Any unique ideas germinating from the above research trends can potentially lead to new discoveries in biochemistry and novel applications in biotechnology. Much like Current Topics in Biochemical Research [24], Biochemistry and Analytical Biochemistry is also an international journal publishing original articles and review articles in all areas of biochemistry. All readers are strongly encouraged to report their newly developed analytical methods and techniques for rapid dissemination to the scientific community. Our journal aims to provide free and unrestricted access of scientific knowledge via the Internet as a means of accelerating scientific discovery [25].

\section{References}

1. Elmazar MM, El-Abhar HS, Schaalan MF, Farag NA (2013) Phytol/Phytanic acid and insulin resistance: potential role of phytanic acid proven by docking simulation and modulation of biochemical alterations. PLoS One 8: e45638.

2. Mu Q, Hu T, Yu J (2013) Molecular insight into the steric shielding effect of PEG on the conjugated staphylokinase: biochemical characterization and molecular dynamics simulation. PLoS One 8: e68559.

3. Mitchell R, Charlwood C, Thomas SD, Bellis M, Langlois NEI. (2013) An audit of the contribution to post-mortem examination diagnosis of individual analyte results obtained from biochemical. Forensic Sci Med Pathol 9: 515-520.

4. Kespohl S, Maryska S, Zahradnik E, Sander I, Brüning T, et al. (2013) Biochemical and immunological analysis of mould skin prick test solution current status of standardization. Clin Exp Allergy 43: 1286-1296.

5. Kamdem I, Hiligsmann S, Vanderghem C, Bilik I, Paquot M, et al. (2013) Comparative biochemical analysis during the anaerobic digestion of lignocellulosic biomass from six morphological parts of Williams Cavendish banana (Triploid Musa AAA group) plants. World J Microbiol Biotechnol 29 : 2259-2270.

6. Hansen MAT, Jørgensen H, Laursen KH, Schjoerring JK, Felby C (2013) Structural and chemical analysis of process residue from biochemical conversion of wheat straw ( Triticum aestivum L.) to ethanol. Biomass \& Bioenergy 56: 572-581.

7. Kang J, Kim K, Oh G, Rhee S (2013) Analysis on biochemical methane potential of agricultural byproducts with different types of silage storage. J Mater Cycles Waste Manag.

8. Ahmad MA, Gupta M (2013) Exposure of Brassica juncea (L) to arsenic species in hydroponic medium: comparative analysis in accumulation and biochemical and transcriptional alterations. Environ Sci Pollut Res Int 20: 8141-8150.

9. Apostel C, Dippold M, Glaser B, Kuzyakov Y (2013) Biochemical pathways of amino acids in soil: assessment by position-specific labeling and 13 C-PLFA analysis. Soil Biology and Biochemistry 67: 31-40.

10. Geisler K, Hughes RK, Sainsbury F, Lomonossoff GP, Rejzek M, et al. (2013) Biochemical analysis of a multifunctional cytochrome P450 (CYP51) enzyme required for synthesis of antimicrobial triterpenes in plants. Proc Natl Acad Sci U S A 110: E3360-3367.

11. Martiniuk F, Reggi S, Tchou-Wong KM, Rom WN, Busconi M, et al. (2013) Production of a functional human acid maltase in tobacco seeds: biochemical analysis, uptake by human GSDII cells, and in vivo studies in GAA knockout mice. Appl Biochem Biotechnol 171: 916-926.

12. Mikashinovich ZI, Belousova ES (2013) Analysis of Biochemical Changes in 
Rat Erythrocytes Induced by Long-Term Simvastatin Treatment. Bulletin of Experimental Biology and Medicine 155: 576-579.

13. Yokoyama H, Kobayashi D, Takizawa N, Fujii S, Matsui I (2013) Structural and biochemical analysis of a thermostable membrane-bound stomatin-specific protease. J Synchrotron Radiat 20: 933-937.

14. Benavente R, Esteban-Torres M, Acebrón I, de Las Rivas B, Muñoz R, et al. (2013) Structure, biochemical characterization and analysis of the pleomorphism of carboxylesterase Cest-2923 from Lactobacillus plantarum WCFS1. FEBS J.

15. Lee DW, Kang JS, Jung CS, Han HR, Moon YS, et al. (2013) Identification and biochemical analysis of a novel pectate lyase 3 gene in Bursaphelenchus xylophilus. Journal of Asia-Pacific Entomology 16: 335-342.

16. Chen R, Ren S, Meng T, Aguilar J, Sun Y (2013) Impact of Glutathione-STransferases (GST) Polymorphisms and Hypermethylation of Relevant Genes on Risk of Prostate Cancer Biochemical Recurrence: A Meta-Analysis. PLoS One 8: e74775.

17. Galsky MD, Xie W, Nakabayashi M, Ross R W, Fennessy FM, et al. (2013) Analysis of the correlation between endorectal MRI response to neoadjuvant chemotherapy and biochemical recurrence in patients with high-risk localized prostate cancer. Prostate Cancer Prostatic Dis 16: 266-270.

18. Choi S, Kelber J, Jiang X, Strnadel J, Fujimura K, et al. (2013) Procedures for the biochemical enrichment and proteomic analysis of the cytoskeletome. Anal Biochem .

19. Escribano BM, Molina A, Valera M, Tovar P, Agüera El, et al. (2013) Genetic analysis of haematological and plasma biochemical parameters in the Spanish purebred horse exercised on a treadmill. Animal 7: 1414-1422.

20. Pantazis Y, Katsoulakis MA, Vlachos DG (2013) Parametric sensitivity analysis for biochemical reaction networks based on pathwise information theory. BMC Bioinformatics 14: 311

21. Hou S, Sun L, Wieczorek SA, Kalwarczyk T, Kaminski TS, et al. (2014) Fluorescence correlation spectroscopy analysis for accurate determination of proportion of doubly labeled DNA in fluorescent DNA pool for quantitative biochemical assays. Biosensors and Bioelectronics 51: 8-15.

22. Fatima N, Ahmad N, Anis M, Ahmad I (2013) An improved in vitro encapsulation protocol, biochemical analysis and genetic integrity using DNA based molecular markers in regenerated plants of Withania somnifera L. Industrial Crops and Products 50: 468-477.

23. Georgelis N, Nikolaidis N, Cosgrove DJ (2014) Biochemical analysis of expansin-like proteins from microbes. Carbohydr Polym 100: 17-23.

24. Current Topics in Biochemical Research.

25. Journal of Biochemistry \& Analytical Biochemistry. 